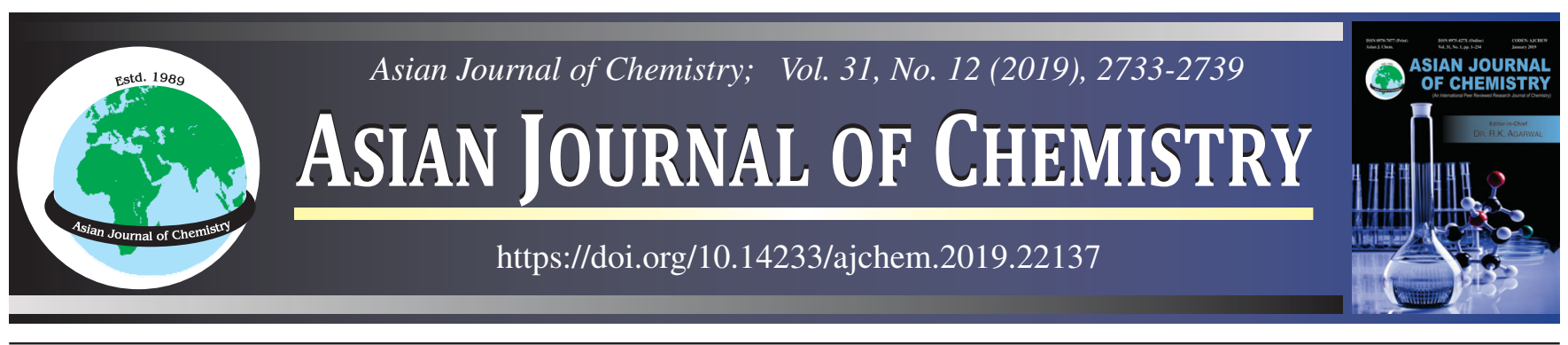

\title{
Design, Synthesis and Molecular Docking Studies of Novel Pyrazole Benzimidazole Derivatives as Potent Antibacterial Agents
}

\section{Srinivasa Rao Dasari ${ }^{1,2, *}$, Subbaiah Tondepu ${ }^{3}$, LaKshmana Rao Vadali $^{2}$ and Nareshvarma SeElam ${ }^{1}$}

${ }^{1}$ Department of Chemistry, Koneru Lakshmaiah Education Foundation, Vaddeswaram, Guntur-522502, India

${ }^{2}$ Mylan Laboratories Ltd, Hyderabad-500049, India

${ }^{3}$ Department of Chemical Engineering, Vignan's Foundation for Science, Technology and Research, Vadlamudi, Guntur-522213, India

*Corresponding author: E-mail: dasari43.srinivas@gmail.com

Received: 16 April 2019;

Accepted: 16 June 2019;

Published online: 16 November 2019;

AJC-19612

A novel series of pyrazole benzimidazole derivatives were synthesized and the structure of the final targets $4 \mathbf{a}-\mathbf{h}$ were confirmed by IR, Mass, ${ }^{13} \mathrm{C}$ NMR and ${ }^{1} \mathrm{H}$ NMR spectral analysis. The new pyrazole core with imidazole and benzimidazoles derivatives were evaluated for in vitro antibacterial, antifungal activity against six bacterial strains significantly. In dispersion, $\mathbf{4 c}, \mathbf{4 f}$ and $\mathbf{4 g}$ had the highest antibacterial activities on these microorganisms Bacillus subtilis B29, Escherichia coli E266, with zone of inhibition 21, 19 and 19 mm, respectively. Compounds $\mathbf{4 a}, \mathbf{4 c}, \mathbf{4 h}$ shows good antifungal activity against $A$. niger, Fusarium oxysporum fungal strains. Further, molecular docking for protein ligands interactions was performed using the crystal structure of C(30) carotenoid dehydrosqualene synthase from Staphylococcus aureus complexed with bisphosphonate BPH-700. Among the final compounds $\mathbf{4 e}, \mathbf{4 g}$ and $\mathbf{4 h}$ show highest binding energy $\Delta \mathrm{G}=-7.89$, -7.48 and $-7.08 \mathrm{Kcal} / \mathrm{mol}$, respectively and amino acid interactions Lys273, Asp27.

Keywords: Imidazole, Pyrazole, Antibacterial activity, Antifungal activity, Molecular modeling, 2 ZCS.

\section{INTRODUCTION}

One of the most complex branches of organic chemistry is the chemistry of heterocyclic composites. It is equally compulsive for its "theoretical implications", "diversified synthetic procedures" and for the "physiological and industrial importance" of heterocyclic compounds [1]. Heterocyclic compounds in particular, will be widely studied for cause of their intrinsic interest as well as many medicines, natural products, dyestuffs and drugs belong to their group [2]. Heterocycles are the cyclic organic compounds with at least one atom other than the carbon atom in their ring system. More than one third of the organic compounds are likely to be heterocycles. Majority of alkaloids, vitamins, synthetic medicines, antibiotics and substances like nucleic acids which are more closely associated with the human life evolution belongs to heterocyclic compounds $[1,2]$.

Pyrazoles exhibit the typical aromatic system properties, which are in fact rather prominent in their structures having insecticide, herbicide properties and pharmaceutical interest as anti-inflammatory and antipyretic. Even though the pyrazoles are very much familiar for 80 years, their chemistry exploration is on slow pace. Pyrazole compounds have the potential biological activities or have shown good pharmacological effects such as, anti-inflammatory [3], antiviral [4], antimicrobial [5], anticonvulsant [6], antitumor [7], fungicidal activities [8] and antihistaminic [9]. The pyrazole moiety has important role in some drugs structure, for example, some aryl pyrazole derivatives have anti-HIV-1 activity [10-12] and some pyrazole3-carboxamide moiety has anti-CB1 cannabinoid ability [13]. Moreover, they are used as anti-inflammotary, antitumor, antipsychotic, antimicrobial and analgesic agents [14]. It is a fact that pyrazole derivatives are an important class of compounds for medicines, biological systems, agrochemicals and many fields of industrial products.

Benzimidazole derivatives are a blend of many varieties of pharmacodynamic and pharmacokinetic characteristics. Benzimidazoles belong to the most bioactive heterocyclic group that demonstrates a wide range of biological activities exclusively as a vitamin $B_{12}$ constituent [15]. The pharmacolo-

This is an open access journal, and articles are distributed under the terms of the Attribution 4.0 International (CC BY 4.0) License. This license lets others distribute, remix, tweak, and build upon your work, even commercially, as long as they credit the author for the original creation. You must give appropriate credit, provide a link to the license, and indicate if changes were made. 
gical properties of benzimidazoles containing mebendazole, albendazole and thiabendazole moieties have been well documented and extensively used as anthelmintic drugs [16]. Moreover, benzimidazole have natural abundance of nucleotide structural entity and thus it can interacts more easily with biopolymers of the living system. This nature leads to its enormous biological aspects like antihelminthic [17], antifungal [18], antiallergic [19], antimicrobial [20,21], antiviral [22] and antineoplastic activities [23]. The inclusion of benzimidazole nucleus became the vital synthetic strategy for drug discovery, biological studies and many applications of crop protection chemistry as the proteinase is being associated to a majority of disease states like inflammation, thrombosis, tumor growth, bronchoconstiction and invasion [24].

However, pyrazole core with benzimidazoles, imidazole are rarely found in nature and that makes the pyrazole synthesis crucial. In this view, we report the synthesis of novel $\mathrm{N}$-alkyl imidazole pyrazole derivatives (4a-h) incorporated with 2-butyl4-chloro- $1 \mathrm{H}$-imidazole-5-carbaldehyde and acetophenone/2acetyl benzimidazole which obsessed a broad range of biological activity cheering to antibacterial activity against Gram-positive, Gram-negative organisms. Additionally, we also report the anti- fungal activity against two microorganisms. For molecular docking, it was found that the target compounds has potential to inhibit nitrate reductase protein (PDB code, 2ZCS) which showed kinetic energies and good affinity by using auto-dock 4.2 software. By comparing the free energies equivalent to binding of title compounds with target protein, it has been revealed that most of the compounds interact with receptors. Almost all molecules are exhibiting lower free energy values, indicating more thermodynamically favoured interaction. Therefore, these lead molecules are synthesizing, exhibit better activity to improve further efficacy and specificity as a potent antibacterial and antifungal agents.

\section{EXPERIMENTAL}

This work deals with the synthesis and characterization of novel $\mathrm{N}$-alkyl imidazole pyrazole derivatives $\mathbf{4 a - h}$ starting with 2-butyl-4-chloro- $1 H$-imidazole-5-carbaldehyde (1). Initially $n$-alkyl-2-butyl-4-chloro- $1 H$-imidazole-5-carbaldehydes (2a-d) reacts with acetophenone/2-acetyl benzimdazole in $20 \%$ sodium hydroxide solution to give $\mathbf{3 a}-\mathbf{h}$. The resulted compounds cyclized with hydrazine hydrate in methanol to give the desired products $\mathbf{4 a - h}$ showed in Scheme-I. The synthe-<smiles>[R8]C(=O)/C=C/c1c(Cl)nc(CCCC)n1[R]</smiles>

1

2a-d

3a-h

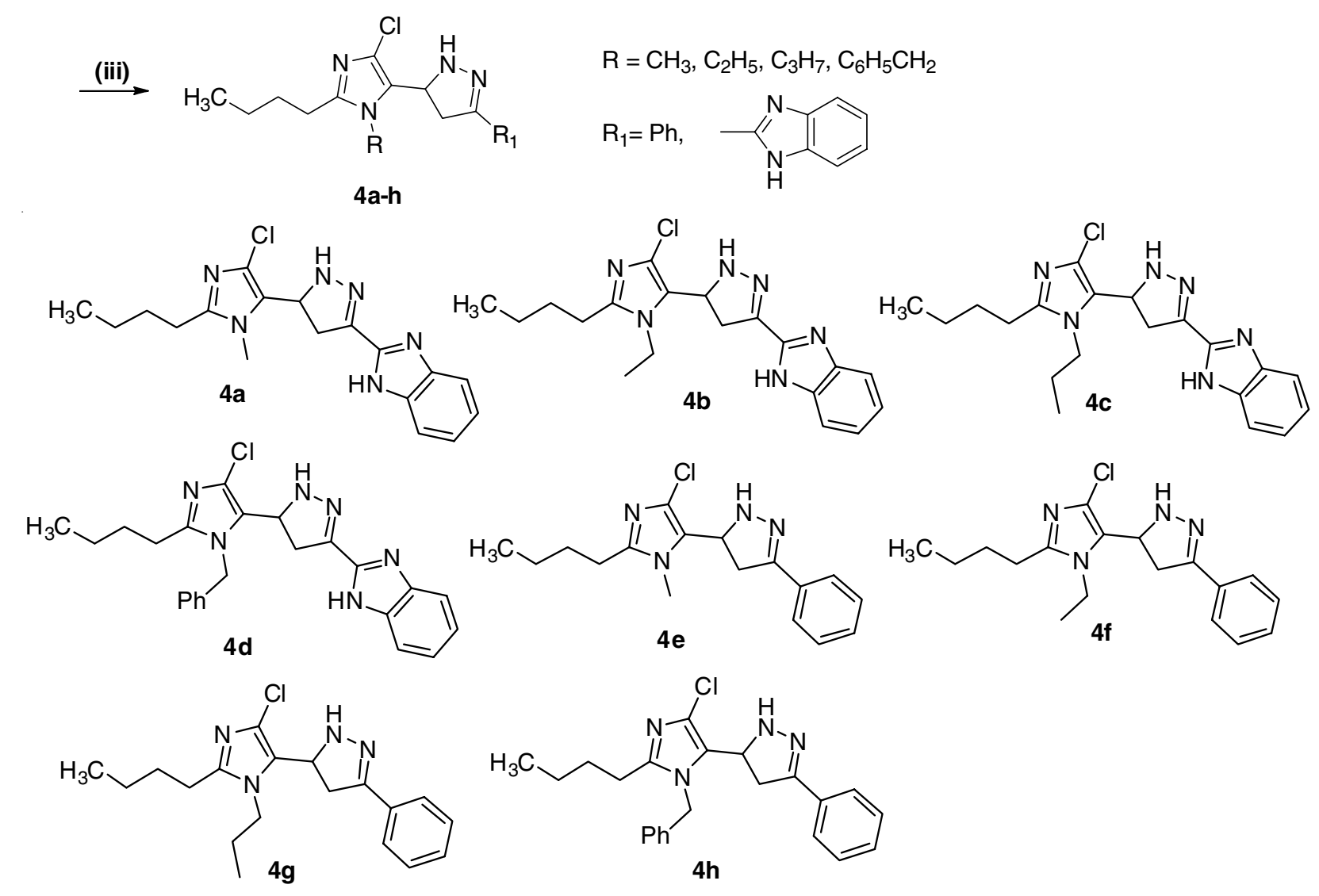

Scheme-I: Synthesis of novel pyrazole benzimidazole derivatives $\mathbf{4 a - h}$; Reagent and conditions: (i) PEG, alkylating agent, $50{ }^{\circ} \mathrm{C}, 15$ min, 85 $\%$ (ii) acetophenone/2-acetyl benzimdazole, $20 \%$ aq. $\mathrm{NaOH}$, room temperature, 7-9 h, 72-86 \% (iii) $\mathrm{NH}_{2} \mathrm{NH}_{2}$, acetic acid, methanol, $74-89 \%$ 
sized compounds structures were confirmed by FT-IR, ${ }^{1} \mathrm{H}$ NMR ${ }^{13} \mathrm{C}$ NMR, elemental and mass spectral analysis.

General procedure for the preparation of $E$-imidazolepropene-1-one intermediates (3a-h): A solution of 2-butyl4-chloro- $1 H$-imidazole-5-carbaldehyde (1) $(5 \mathrm{~g}, 26 \mathrm{mmol})$, is added with corresponding 2 -acetyl benzimdazole ( $4.3 \mathrm{~g}, 26$ $\mathrm{mmol})$, acetophenone $(3.3 \mathrm{~g}, 27 \mathrm{mmol})$ in $20 \%$ sodium hydroxide solution $(10 \mathrm{~mL})$ and methanol $(5 \mathrm{~mL})$ at ambient temperature and stirred for about 7-9 h. When the reaction completed as shown by TLC analysis by using EtOAc:hexane as mobile phase, the reaction mass was poured into ice water $(50 \mathrm{~mL})$ to get the product precipitated out as a solid. It was then filtered and dried to obtain the corresponding imidazolepropene-1-one intermediates $\mathbf{3 a}-\mathbf{h}$ with good yield.

(E)-1-(1H-Benzo[d]imidazol-2-yl)-3-(2-butyl-4-chloro1-methyl-1H-imidazol-5-yl)prop-2-en-1-one (3a): Yield 84 $\%$; Off-white solid, m.p. $178-179^{\circ} \mathrm{C}$; Elemental analysis calcd. (found) \% of $\mathrm{C}_{18} \mathrm{H}_{19} \mathrm{~N}_{4} \mathrm{OCl}$ : C, 63.06 (63.90); H, 5.59 (6.23); $\mathrm{Cl}, 10.34$ (10.01); N, 16.34 (15.44). IR (KBr, $\left.v_{\max }, \mathrm{cm}^{-1}\right): 824$ (CCl str.), 1605, 1554 (Ar-C=C str.), 1760 (CO str.), 2846, 2926 (CH str.), 3052 (=CH str.), 3420 (NH str.); ${ }^{1} \mathrm{H}$ NMR (400 $\mathrm{MHz}, \mathrm{DMSO} / \mathrm{TMS}): \delta_{\mathrm{ppm}} 1.08\left(\mathrm{t}, 3 \mathrm{H}, J=6.2 \mathrm{~Hz}, \mathrm{CH}_{3}\right), 1.28-$ 1.36 (sex, 2H, $\mathrm{CH}_{2}$ ), 1.67-1.68 (quin, 2H, $\mathrm{CH}_{2}$ ), 2.68-2.69 (t, $\left.2 \mathrm{H}, J=6.5 \mathrm{~Hz}, \mathrm{CH}_{2}\right), 3.8\left(\mathrm{~s}, 3 \mathrm{H}, \mathrm{NCH}_{3}\right), 7.37-7.39(\mathrm{~m}, 2 \mathrm{H}$, vinyl proton \& $\mathrm{ArH}), 7.66-7.74(\mathrm{~m}, 3 \mathrm{H}, \mathrm{ArH}), 7.84-7.88(\mathrm{~d}$, $1 \mathrm{H}, J=7.0 \mathrm{~Hz}$, vinyl proton).

(E)-1-(1H-Benzo[d] imidazol-2-yl)-3-(2-butyl-4-chloro1-ethyl-1H-imidazol-5-yl)prop-2-en-1-one (3b): Yield $85 \%$; Off-white solid, m.p. $184-187^{\circ} \mathrm{C}$; Elemental analysis calcd. (found) \% of $\mathrm{C}_{19} \mathrm{H}_{21} \mathrm{~N}_{4} \mathrm{OCl}$ : C, 63.95 (64.75); $\mathrm{H}, 5.93$ (6.83); $\mathrm{Cl}, 9.94$ (9.54); N, 15.70 (14.82). IR ( $\left.\mathrm{KBr}, \mathrm{v}_{\max }, \mathrm{cm}^{-1}\right)$ : 832 (CCl str.), 1604, 1524 (Ar-C=C str.), 1760 (CO str.), 2915, 2962 (CH str.), 3065 (=CH str.), 3305 (NH str.); ${ }^{1} \mathrm{H}$ NMR $(400 \mathrm{MHz}$, DMSO/TMS): $\delta_{\mathrm{ppm}} 1.15\left(\mathrm{t}, 3 \mathrm{H}, J=6.5 \mathrm{~Hz}, \mathrm{CH}_{3}\right), 1.29-1.35$ (sex, 2H, $\mathrm{CH}_{2}$ ), 1.62-1.68 (quin, $2 \mathrm{H}, \mathrm{CH}_{2}$ ), 2.06 (t, 3H, $J=7.0$ $\mathrm{Hz}, \mathrm{NCH}_{2} \mathrm{CH}_{3}$ ), 2.64-2.67 (t, $\left.2 \mathrm{H}, J=6.5 \mathrm{~Hz}, \mathrm{CH}_{2}\right), 4.14$ (q, $\left.2 \mathrm{H}, J=7.5 \mathrm{~Hz}, \mathrm{NCH}_{2}\right), 7.34-7.36$ ( $\mathrm{m}, 2 \mathrm{H}$, vinyl proton \& $\mathrm{ArH}$ ), 7.61-7.69 (m, 3H, ArH), 7.89-7.93 (d, $1 \mathrm{H}, J=7.0 \mathrm{~Hz}$, vinyl proton).

(E)-1-(1H-Benzo[d] imidazol-2-yl)-3-(2-butyl-4-chloro1-propyl-1 $H$-imidazol-5-yl)prop-2-en-1-one (3c): Yield 83 $\%$; Off-white solid, m.p. $184-187^{\circ} \mathrm{C}$; Elemental analysis calcd. (found) \% of $\mathrm{C}_{20} \mathrm{H}_{23} \mathrm{~N}_{4} \mathrm{OCl}$ : C, 64.77 (65.69); H, 6.25 (6.95); $\mathrm{Cl}, 9.56$ (9.06); N, 15.11 (14.05). IR ( $\left.\mathrm{KBr}, \mathrm{v}_{\max }, \mathrm{cm}^{-1}\right): 845$ (CCl str.), 1621, 1546 (Ar-C=C str.), 1780 (CO str.), 2893, 2962 (CH str.), 3062 (=CH str.), 3325 (NH str.); ${ }^{1} \mathrm{H}$ NMR $(400 \mathrm{MHz}$, DMSO/TMS): $\delta_{\mathrm{ppm}} 0.88\left(\mathrm{t}, 3 \mathrm{H}, J=7.0 \mathrm{~Hz}, \mathrm{CH}_{3}\right), 1.26-1.34$ (sex, 2H, $\mathrm{CH}_{2}$ ), 1.57-1.63 (quin, $2 \mathrm{H}, \mathrm{CH}_{2}$ ), 2.58-2.69 (t, $2 \mathrm{H}, \mathrm{J}$ $\left.=7.0 \mathrm{~Hz}, \mathrm{CH}_{2}\right), 2.9\left(\mathrm{t}, 3 \mathrm{H}, J=6.5 \mathrm{~Hz}, \mathrm{CH}_{3}\right), 7.47-7.52(\mathrm{~m}$, $2 \mathrm{H}$, vinyl proton \& $\mathrm{ArH}), 7.72-7.76(\mathrm{~m}, 3 \mathrm{H}, \mathrm{ArH}), 7.94-7.98$ (d, $1 \mathrm{H}, J=6.8 \mathrm{~Hz}$, vinyl proton), LC-MS: $m / z: 407(\mathrm{M}+1)^{+}$.

(E)-1-(1H-Benzo[d] imidazol-2-yl)-3-(1-benzyl-2-butyl4-chloro-1H-imidazol-5-yl)prop-2-en-1-one (3d): Yield 85 $\%$; Pale yellow solid, m.p. $189-195{ }^{\circ} \mathrm{C}$; Elemental analysis calcd. (found) $\%$ of $\mathrm{C}_{20} \mathrm{H}_{23} \mathrm{~N}_{4} \mathrm{OCl}$ : C, 68.81 (68.75); $\mathrm{H}, 5.53$ (5.83); $\mathrm{Cl}, 8.46$ (8.26); N, 13.37 (13.19). IR (KBr, $\left.v_{\max }, \mathrm{cm}^{-1}\right)$ : 841 (CCl str.), 1622, 1578 (Ar-C=C str.), 1795 (CO str.), 2890, 2955 (CH str.), 3054 (=CH str.), 3250 (NH str.); ${ }^{1} \mathrm{H}$ NMR (400
MHz, DMSO/TMS): $\delta_{\mathrm{ppm}} 0.89$ (t, 3H, $\left.J=6.8 \mathrm{~Hz}, \mathrm{CH}_{3}\right), 1.30-$ 1.36 (sex, $2 \mathrm{H}, \mathrm{CH}_{2}$ ), 1.62-1.70 (quin, $2 \mathrm{H}, \mathrm{CH}_{2}$ ), 2.65-2.69 (t, $\left.2 \mathrm{H}, \mathrm{CH}_{2}\right), 7.4-7.5$ (d, $1 \mathrm{H}$, vinyl proton), 7.59-7.67 (m, 4H, $\mathrm{ArH}$ ), $8.0(\mathrm{~m}, 2 \mathrm{H}$, vinyl proton \& ArH), 12.84 (s, $1 \mathrm{H}, \mathrm{NH})$. LC-MS: $m / z: 289(\mathrm{M}+1)^{+}$.

(E)-3-(2-Butyl-4-chloro-1-methyl-1H-imidazol-5-yl)-1phenylprop-2-en-1-one (3e): Yield 85\%; Off-white solid, m.p. $189-195^{\circ} \mathrm{C}$; Elemental analysis calcd. (found) $\%$ of $\mathrm{C}_{17} \mathrm{H}_{19} \mathrm{~N}_{2} \mathrm{OCl}$ : C 67.43 (67.25); H, 6.32 (6.91); Cl, 11.71 (11.67); N, 9.25 (9.02). IR (KBr, $\left.v_{\max }, \mathrm{cm}^{-1}\right)$ : 825 (CCl str.), 1608, 1554 (Ar$\mathrm{C}=\mathrm{C}$ str.), 1785 (CO str.), 2885, 2951 (CH str.), $3051(=\mathrm{CH}$ str.); ${ }^{1} \mathrm{H}$ NMR (400 MHz, DMSO/TMS): $\delta_{\mathrm{ppm}} 1.22$ (t, 3H, $J=$ 7.0 Hz, $\mathrm{CH}_{3}$ ), 1.29-1.34 (sex, $\left.2 \mathrm{H}, \mathrm{CH}_{2}\right), 1.64-1.72$ (quin, $2 \mathrm{H}$, $\left.\mathrm{CH}_{2}\right), 2.64-2.69\left(\mathrm{t}, 2 \mathrm{H}, \mathrm{CH}_{2}\right), 7.34-7.35$ (d, $1 \mathrm{H}$, vinyl proton), 7.62-7.69 (m, $4 \mathrm{H}, \mathrm{ArH}), 8.20-8.25(\mathrm{~m}, 2 \mathrm{H}$, vinyl proton \& ArH); LC-MS: $m / z: 289(\mathrm{M}+1)^{+}$.

General procedure for the preparation of imidazole containing pyrazole compounds 4a-h: A solution of $3 \mathbf{a}(5 \mathrm{~g}$, $17 \mathrm{mmol})$ in methanol $(30 \mathrm{~mL})$ was added with hydrazine hydrate $(1 \mathrm{~g}, 20 \mathrm{mmol})$, acetic acid $(0.2 \mathrm{~mL})$ and the mixture was heated to reflux temperature, stirred for about $3 \mathrm{~h}$ till the completion of the starting materials. After the reaction got completed, it was cooled to room temperature and added into ice cold water $(50 \mathrm{~mL})$. The precipitated solid was filtered and washed twice with chilled water $(2 \times 20 \mathrm{~mL})$ and dried to get 2-(5-(2-butyl-4-chloro-1-methyl-1 $H$-imidazol-5-yl)-4,5dihydro- $1 H$-pyrazol-3-yl)-1 $H$-benzo[ $d]$ imidazole (4a). The remaining targets were prepared same as above procedure with different quantities for desired products.

2-(5-(2-Butyl-4-chloro-1-methyl-1H-imidazol-5-yl)4,5-dihydro-1 $H$-pyrazol-3-yl)-1 $H$-benzo $[d]$ imidazole (4a): Yield $(4.1 \mathrm{~g}, 79 \%)$; Off-white solid, m.p. $202-203{ }^{\circ} \mathrm{C}$; Elemental analysis calcd. (found) $\%$ of $\mathrm{C}_{18} \mathrm{H}_{21} \mathrm{~N}_{6} \mathrm{Cl}$ : C, 60.58 (61.53); H, 5.93 (6.25); Cl, 9.93 (9.56); N, 23.55 (22.66). IR (KBr, $v_{\max }$, $\left.\mathrm{cm}^{-1}\right): 857$ (CCl str.), 1392 (C-N str.), 1452 (C=N str.), 1608, 1505 (Ar-C=C str.), 1739 (CO str.), 2926, 2952 (CH str.), 3092 (=CH str.), 3260 (NH str.); ${ }^{1} \mathrm{H}$ NMR (400 MHz, $\left.\mathrm{CDCl}_{3} / \mathrm{TMS}\right)$ : $\delta_{\mathrm{ppm}} 1.13\left(\mathrm{t}, 3 \mathrm{H}, J=6.5 \mathrm{~Hz}, \mathrm{CH}_{3}\right), 1.28-1.31$ (sex, $2 \mathrm{H}, \mathrm{CH}_{2}$ ), 1.41-1.58 (quin, $2 \mathrm{H}, \mathrm{CH}_{2}$ ), 2.66 (t, $2 \mathrm{H}, J=6.5 \mathrm{~Hz}, \mathrm{CH}_{2}$ ), 3.58 (d, $2 \mathrm{H}, J=7.0 \mathrm{~Hz}$, pyrazol proton), 3.78 (s, $\left.3 \mathrm{H}, \mathrm{NCH}_{3}\right), 5.17$ (t, $1 \mathrm{H}, J=7.0 \mathrm{~Hz}$, pyrzole proton), $6.16(\mathrm{~s}, 1 \mathrm{H}$, benzimdazole$\mathrm{NH}), 7.26-7.53(\mathrm{~m}, 4 \mathrm{H}, \mathrm{ArH}), 9.33(\mathrm{~s}, 1 \mathrm{H}$, pyrazole- $\mathrm{NH}) ;{ }^{13} \mathrm{C}$ NMR $\left(100 \mathrm{MHz}, \mathrm{DMSO}-d_{6}\right) \delta_{\mathrm{ppm}} 160.00,138.27,136.88$, $131.15,127.77,125.76,124.98,121.67,120.19,115.00,54.83$, 53.63, 48.56, 35.27, 30.71, 23.07. LC-MS: $m / z: 356(\mathrm{M}+1)^{+}$.

2-(5-(2-Butyl-4-chloro-1-ethyl-1H-imidazol-5-yl)-4,5dihydro-1 $H$-pyrazol-3-yl)-1 $H$-benzo $[d]$ imidazole $(4 \mathrm{~b})$ : Yield (85\%); Off-white solid, m.p. 197-198 ${ }^{\circ} \mathrm{C}$; Elemental analysis calcd. (found) $\%$ of $\mathrm{C}_{19} \mathrm{H}_{23} \mathrm{~N}_{6} \mathrm{Cl}: \mathrm{C}, 61.53$ (61.53); $\mathrm{H}$, 6.25 (6.25); Cl, 9.56 (9.56); N, 22.66 (22.66). IR (KBr, $v_{\max }$, $\left.\mathrm{cm}^{-1}\right): 836$ (CCl str.), 1376 (C-N str.), 1458 (C=N str.), 1517, 1610 (Ar-C=C str.), 1658 (CO str.), 2932, 2957 (CH str.), 3071 (=CH str.), 3277 (NH str.); ${ }^{1} \mathrm{H}$ NMR (400 MHz, DMSO/TMS): $\delta_{\mathrm{ppm}} 1.02\left(\mathrm{t}, 3 \mathrm{H}, J=6.5 \mathrm{~Hz}, \mathrm{CH}_{3}\right), 1.23-1.28\left(\mathrm{sex}, 2 \mathrm{H}, \mathrm{CH}_{2}\right)$, 1.42-1.60 (quint, $2 \mathrm{H}, \mathrm{CH}_{2}$ ), 2.60 (t, $2 \mathrm{H}, J=6.5 \mathrm{~Hz}, \mathrm{CH}_{2}$ ), 3.23 (t, $\left.3 \mathrm{H}, J=6.5 \mathrm{~Hz}, \mathrm{NCH}_{2} \mathrm{CH}_{3}\right), 3.56(\mathrm{~d}, 2 \mathrm{H}, J=7.0 \mathrm{~Hz}$, pyrazol proton), 4.23 (t, $\left.2 \mathrm{H}, J=6.5 \mathrm{~Hz}, \mathrm{NCH}_{2}\right), 5.21$ (t, $1 \mathrm{H}, J=6.5$ $\mathrm{Hz}$, pyrzole proton), 6.28 (s, 1H, benzimdazole-NH), 7.29-7.55 
(m, 4H, ArH), $9.38\left(\mathrm{~s}, 1 \mathrm{H}\right.$, pyrazole-NH); ${ }^{13} \mathrm{C}$ NMR $(100 \mathrm{MHz}$, DMSO- $\left.d_{6}\right) \delta_{\mathrm{ppm}} 155.16,147.79,138.19,134.27,132.31$, 127.81, 124.71, 122.94, 121.80, 116.94, 55.72, 45.30, 44.24, 42.85, 34.91, 29.34, 24.83, 21.90. LC-MS: $m / z: 370(\mathrm{M}+1)^{+}$.

2-(5-(2-Butyl-4-chloro-1-propyl-1H-imidazol-5-yl)4,5-dihydro-1 $H$-pyrazol-3-yl)-1H-benzo[d]imidazole (4c): Yield (3.8 g, $74 \%$ ); Off-white solid, m.p. $194-195{ }^{\circ} \mathrm{C}$; Elemental analysis calcd. (found) $\%$ of $\mathrm{C}_{19} \mathrm{H}_{23} \mathrm{~N}_{6} \mathrm{Cl}$ : $\mathrm{C}, 62.41$ (62.35); $\mathrm{H}$, 6.55 (6.50); $\mathrm{Cl}, 9.21$ (9.17); N, 21.83 (21.80). IR (KBr, $v_{\max }$, $\left.\mathrm{cm}^{-1}\right): 853$ (CCl str.), 1378 (C-N str.), 1449 (C=N str.), 1601 (Ar-C=C str.), 1786 (CO str.), 2931, 2955 (CH str.), 3076 (=CH str.), 3290 (NH str.); ${ }^{1} \mathrm{H}$ NMR (400 MHz, $\mathrm{CDCl}_{3} / \mathrm{TMS}$ ): $\delta_{\mathrm{ppm}}$ $0.91\left(\mathrm{t}, 3 \mathrm{H}, J=6.5 \mathrm{~Hz}, \mathrm{CH}_{3}\right), 1.21-1.29$ (sex, $\left.2 \mathrm{H}, \mathrm{CH}_{2}\right), 1.39$ $1.56\left(\mathrm{~m}, 4 \mathrm{H}, \mathrm{CH}_{2}\right), 2.68\left(\mathrm{t}, 2 \mathrm{H}, J=6.5 \mathrm{~Hz}, \mathrm{CH}_{2}\right), 2.78(\mathrm{t}, 3 \mathrm{H}$, $\left.J=6.5 \mathrm{~Hz}, \mathrm{CH}_{3}\right), 3.36(\mathrm{~d}, 1 \mathrm{H}, J=7.0 \mathrm{~Hz}$, pyrazole proton), $3.59(\mathrm{~d}, 2 \mathrm{H}, J=7.0 \mathrm{~Hz}$, pyrazol proton), $5.19(\mathrm{t}, 1 \mathrm{H}$, pyrzole proton), 6.17 (s, 1H, benzimdazole-NH), 7.32-7.54 (m, 4H, Ar$\mathrm{H}), 9.42$ (s, $1 \mathrm{H}$, pyrazole-NH); ${ }^{13} \mathrm{C}$ NMR (100 MHz, DMSO- $d_{6}$ ) $\delta_{\mathrm{ppm}} 155.22,138.40,135.49,132.07,128.28,126.72,125.21$, 121.40, 118.14, 58.73, 52.67, 46.18, 42.84, 36.24, 30.18, 29.31, 28.27, 22.46. LC-MS: $m / z: 384\left(\mathbf{M}^{+}+1\right)$.

2-(5-(1-Benzyl-2-butyl-4-chloro-1H-imidazol-5-yl)-4,5dihydro-1 $\boldsymbol{H}$-pyrazol-3-yl)-1 $\boldsymbol{H}$-benzo[ $\boldsymbol{d}]$ imidazole (4d): Yield (4.7 g, $89 \%$ ); Pale yellow solid, m.p. $174-175^{\circ} \mathrm{C}$; Elemental analysis calcd. (found) $\%$ of $\mathrm{C}_{24} \mathrm{H}_{25} \mathrm{~N}_{6} \mathrm{Cl}: \mathrm{C}, 66.58$ (66.42); $\mathrm{H}$, 5.82 (5.71); $\mathrm{Cl}, 8.19$ (8.20); N, 19.41 (19.24). IR (KBr, $v_{\max }$, $\left.\mathrm{cm}^{-1}\right): 836$ (CCl str.), 1392 (C-N str.), $1456(\mathrm{C}=\mathrm{N}$ str.), 1612 (Ar-C=C str.), 2856, 2942 (CH str.), 3052 (=CH str.), 3320 (NH str.); ${ }^{1} \mathrm{H}$ NMR (400 MHz, $\mathrm{CDCl}_{3} / \mathrm{TMS}$ ): $\delta_{\mathrm{ppm}} 1.05$ (t, $3 \mathrm{H}$, $J=6.5 \mathrm{~Hz}, \mathrm{CH}_{3}$ ), $1.32-1.37$ (sex, $2 \mathrm{H}, \mathrm{CH}_{2}$ ), 1.46-1.57 (quin, $\left.2 \mathrm{H}, \mathrm{CH}_{2}\right), 2.72-2.79\left(\mathrm{t}, 2 \mathrm{H}, J=6.5 \mathrm{~Hz}, \mathrm{CH}_{2}\right), 3.52(\mathrm{t}, 1 \mathrm{H}, J=$ $6.5 \mathrm{~Hz}$, pyrazole proton), $3.66(\mathrm{~d}, 2 \mathrm{H}, J=6.5 \mathrm{~Hz}$, pyrazol proton), 4.98 (q, $\left.2 \mathrm{H}, J=7.0 \mathrm{~Hz}, \mathrm{NCH}_{2}\right), 7.42-7.61(\mathrm{~m}, 5 \mathrm{H}$, ArH), 8.12-8.31 (m, 4H, ArH), 8.91 (s, 1H, pyrazole-NH), 11.26 (s, $1 \mathrm{H}$, imdazole proton); ${ }^{13} \mathrm{C}$ NMR (100 MHz, DMSO- $d_{6}$ ) $\delta_{\mathrm{ppm}} 160.18,137.44,135.84,132.77,130.86,129.79,126.83$, 124.22, 122.78, 120.82, 119.34, 115.99, 109.37, 53.64, 48.15, 34.28, 32.74, 29.38, 25.37, 22.31. LC-MS: $m / z 432(\mathrm{M}+1)^{+}$.

5-(2-Butyl-4-chloro-1-methyl-1H-imidazol-5-yl)-3phenyl-4,5-dihydro-1 $\boldsymbol{H}$-pyrazole (4e): Yield (3.9 g, $74 \%$ ); Off-white solid, m.p. $192-193{ }^{\circ} \mathrm{C}$; Elemental analysis calcd. (found) \% of $\mathrm{C}_{17} \mathrm{H}_{21} \mathrm{~N}_{4} \mathrm{Cl}$ : C, 64.45 (64.24); H, 6.68 (6.72); $\mathrm{Cl}, 11.19$ (11.42); N, 17.68 (17.91). IR (KBr, $\left.v_{\max }, \mathrm{cm}^{-1}\right): 848$ (CCl str.), 1368 (C-N str.), 1460 (C=N str.), 1612, 1564 (Ar$\mathrm{C}=\mathrm{C}$ str. $), 2945$ (CH str.), 3050 (=CH str. $), 3325(\mathrm{NH}$ str. $) ;{ }^{1} \mathrm{H}$ NMR (400 MHz, CDCl $/$ TMS): $\delta_{\text {ppm }} 1.09$ (t, $3 \mathrm{H}, J=6.5 \mathrm{~Hz}$, $\mathrm{CH}_{3}$ ), 1.28-1.34 (sex, 2H, $\mathrm{CH}_{2}$ ), 1.62-1.69 (quin, $2 \mathrm{H}, \mathrm{CH}_{2}$ ), $2.84\left(\mathrm{t}, 2 \mathrm{H}, J=6.5 \mathrm{~Hz}, \mathrm{CH}_{2}\right), 3.43(\mathrm{t}, 1 \mathrm{H}, J=6.5 \mathrm{~Hz}$, pyrazole proton), 3.62 (d, $2 \mathrm{H}, J=7.0 \mathrm{~Hz}$, pyrazol proton), 3.79 (s, $3 \mathrm{H}$, $\left.\mathrm{NCH}_{3}\right)$, 7.32-7.49 (m, 3H, Ar-H), 7.93-8.02 (m, 2H, Ar-H), 9.52 (s, $1 \mathrm{H}$, pyrazole-NH); ${ }^{13} \mathrm{C}$ NMR $\left(100 \mathrm{MHz}\right.$, DMSO- $\left.d_{6}\right)$ $\delta_{\mathrm{ppm}} 154.16,138.40,135.49,132.07,128.28,126.72,125.21$, 121.70, 119.14, 59.78, 53.64, 48.15, 42.81, 32.52, 29.38, 25.37, 20.39. LC-MS: $m / z: 316(\mathrm{M}+1)^{+}$.

5-(2-Butyl-4-chloro-1-ethyl-1H-imidazol-5-yl)-3phenyl-4,5-dihydro-1H-pyrazole (4f): Yield (3.8 g, $74 \%$ ); Off-white solid, m.p. $164-165^{\circ} \mathrm{C}$; Elemental analysis calcd. (found) $\%$ of $\mathrm{C}_{17} \mathrm{H}_{21} \mathrm{~N}_{4} \mathrm{Cl}$ : C, 65.34 (65.99); H, 7.01 (7.62);
$\mathrm{Cl}, 10.72$ (10.22); $\mathrm{N}, 16.93$ (16.53). IR (KBr, $\left.v_{\max }, \mathrm{cm}^{-1}\right): 836$ (CCl str.), 1368 (C-N str.), 1452 (C=N str.), 1612, 1536 (Ar$\mathrm{C}=\mathrm{C}$ str. $), 2922,2965(\mathrm{CH}$ str. $), 3072(=\mathrm{CH}$ str. $), 3310(\mathrm{NH}$ str.); ${ }^{1} \mathrm{H}$ NMR (400 MHz, $\mathrm{CDCl}_{3} / \mathrm{TMS}$ ): $\delta_{\mathrm{ppm}} 0.96$ (t, 3H, $J=$ $\left.6.5 \mathrm{~Hz}, \mathrm{CH}_{3}\right), 1.29-1.31$ (sex, $\left.2 \mathrm{H}, \mathrm{CH}_{2}\right), 1.52-1.59\left(\mathrm{~m}, 4 \mathrm{H}, \mathrm{CH}_{2}\right.$ ), $2.67\left(\mathrm{t}, 2 \mathrm{H}, J=6.5 \mathrm{~Hz}, \mathrm{CH}_{2}\right), 2.87$ (t, $3 \mathrm{H}, J=6.5 \mathrm{~Hz}, \mathrm{CH}_{3}$ ), $3.73(\mathrm{~d}, 2 \mathrm{H}, J=7.0 \mathrm{~Hz}$, pyrazole proton), 3.79 (t, $1 \mathrm{H}, J=6.5$ $\mathrm{Hz}$, pyrazol proton), 4.19 (q, $\left.2 \mathrm{H}, J=7.0 \mathrm{~Hz}, \mathrm{NCH}_{2}\right), 7.53-7.66$ (m, 3H, Ar-H), 8.02 (d, 2H, J = 7.0 Hz, Ar-H), $9.46(\mathrm{~s}, 1 \mathrm{H}$, pyrazole-NH); ${ }^{13} \mathrm{C}$ NMR $\left(100 \mathrm{MHz}, \mathrm{DMSO}-d_{6}\right) \delta_{\mathrm{ppm}} 155.63$, $138.38,136.87,131.00,127.80,125.77,121.66,120.16,54.86$, 48.56, 42.01, 30.24, 28.33, 20.71, 14.04. LC-MS: $\mathrm{m} / z: 384$ $\left(\mathrm{M}^{+}+1\right)$.

5-(2-Butyl-4-chloro-1-propyl-1H-imidazol-5-yl)-3phenyl-4,5-dihydro-1H-pyrazole (4g): Yield (3.8 g, $74 \%$ ); Off-white solid, m.p. $142-143{ }^{\circ} \mathrm{C}$; Elemental analysis calcd. (found) $\%$ of $\mathrm{C}_{19} \mathrm{H}_{25} \mathrm{~N}_{4} \mathrm{Cl}$ : C, 66.17 (65.17); $\mathrm{H}, 7.31$ (7.72); $\mathrm{Cl}, 10.28$ (10.46); N, 16.25 (16.18). IR (KBr, $\left.v_{\max }, \mathrm{cm}^{-1}\right): 848$ (CCl str.), 1345 (C-N str.), 1473 (C=N str.), 1584 ( $\mathrm{Ar}-\mathrm{C}=\mathrm{C}$ str.), 2960 (CH str.), 3061 (=CH str.), 3312 (NH str.); ${ }^{1} \mathrm{H}$ NMR (400 MHz, $\left.\mathrm{CDCl}_{3} / \mathrm{TMS}\right): \delta_{\mathrm{ppm}} 1.05\left(\mathrm{t}, 6 \mathrm{H}, J=6.5 \mathrm{~Hz}, \mathrm{CH}_{3}\right.$ ), 1.25 (sex, 2H, $\left.\mathrm{CH}_{2}\right), 1.89$ (m, 4H, $\left.\mathrm{CH}_{2}\right), 2.54(\mathrm{t}, 2 \mathrm{H}, J=6.5$ $\mathrm{Hz}, \mathrm{CH}_{2}$ ), 3.62 (d, $2 \mathrm{H}, J=7.0 \mathrm{~Hz}$, pyrazole proton), 3.88 (t, $1 \mathrm{H}, J=6.5 \mathrm{~Hz}$, pyrazol proton), $4.19\left(\mathrm{t}, 2 \mathrm{H}, J=6.5 \mathrm{~Hz}, \mathrm{NCH}_{2}\right.$ ), 7.28-7.37 (m, 3H, Ar-H), 7.92-7.99 (d, 2H, J=7.0 Hz, Ar-H), $9.62\left(\mathrm{~s}, 1 \mathrm{H}\right.$, pyrazole-NH); ${ }^{13} \mathrm{CNMR}\left(100 \mathrm{MHz}, \mathrm{CDCl}_{3}\right): \delta 154.12$, $138.28,133.15,131.20,130.81,128.60,127.15,126.48,52.6$, 36.12, 33.89, 28.3, 25.37, 21.2, 16.39. LC-MS: $m / z: 345\left(\mathbf{M}^{+}+1\right)$.

5-(1-Benzyl-2-butyl-4-chloro-1H-imidazol-5-yl)-3phenyl-4,5-dihydro-1H-pyrazole (4h): Yield (4.7 g, $89 \%$ ); Off-white solid, m.p. $147-148{ }^{\circ} \mathrm{C}$; Elemental analysis calcd. (found) \% of $\mathrm{C}_{19} \mathrm{H}_{25} \mathrm{~N}_{4} \mathrm{Cl}$ : C, 70.31 (71.15); $\mathrm{H}, 6.41$ (6.28); $\mathrm{Cl}, 9.02$ (9.19); N, 14.26 (14.18). IR (KBr, $\left.v_{\max }, \mathrm{cm}^{-1}\right): 860$ (CCl str.), 1398 (C-N str.), 1462 (C=N str.), 1605 (Ar-C=C str.), 2860, 2945 (CH str.), 3058 (=CH str.), $3321(\mathrm{NH}$ str. $) ;{ }^{1} \mathrm{H}$ NMR (400 MHz, CDCl $3 / \mathrm{TMS}$ ): $\delta_{\mathrm{ppm}} 1.12(\mathrm{t}, 3 \mathrm{H}, J=6.5 \mathrm{~Hz}$, $\mathrm{CH}_{3}$ ), 1.38-1.42 (sex, 2H, $\mathrm{CH}_{2}$ ), 1.56-1.63 (quin, $2 \mathrm{H}, \mathrm{CH}_{2}$ ), $2.65\left(\mathrm{t}, 2 \mathrm{H}, J=6.5 \mathrm{~Hz}, \mathrm{CH}_{2}\right), 3.52(\mathrm{t}, 1 \mathrm{H}, J=6.5 \mathrm{~Hz}$, pyrazole proton), 3.86 (d, 2H, $J=6.5 \mathrm{~Hz}$, pyrazol proton), 4.92 (q, $2 \mathrm{H}$, $\left.J=7.0 \mathrm{~Hz}, \mathrm{NCH}_{2}\right), 7.42-7.58(\mathrm{~m}, 4 \mathrm{H}, \mathrm{ArH}), 7.91-7.99(\mathrm{~d}, 2 \mathrm{H}$, $J=6.5 \mathrm{~Hz}, \mathrm{ArH}), 8.18-8.29(\mathrm{~m}, 4 \mathrm{H}, \mathrm{ArH}), 9.15(\mathrm{~s}, 1 \mathrm{H}$, pyrazole$\mathrm{NH}) ;{ }^{13} \mathrm{C}$ NMR $\left(100 \mathrm{MHz}, \mathrm{DMSO}-d_{6}\right) \delta_{\mathrm{ppm}} 161.02,147.61$, $138.22,133.80,132.71,131.15,129.34,128.46,127.71$, 126.37, 124.91, 121.94, 120.43, 116.43, 48.42, 36.45, 32.16, 29.37, 24.82, 20.91, 18.62. LC-MS: $\mathrm{m} / z 393(\mathrm{M}+1)^{+}$.

Biological evaluation: By using streptomycin $(100 \mu \mathrm{g} /$ $\mathrm{mL}$ ) as a reference antibacterial agent, all the final compounds were tested for in vitro antibacterial activity against three Grampositive strains and three Gram-negative bacteria by adopting cup plate agar diffusion method [25]. The sterilized mediums [1 mg/mL] (autoclaved at $110{ }^{\circ} \mathrm{C}$ for $45 \mathrm{~min}$ ) was inoculated with suspensions of the microorganisms $\left(10^{5} \mathrm{cfu} \mathrm{mL}^{-1}\right)$ as harmonized to McFarland barium sulphate solution and filled into petri-dishes to get a depth of 3-4 mm. For about 18 to 24 $\mathrm{h}$, the mediums were solidified, inverted and incubated at 30$37^{\circ} \mathrm{C}$ till the adequate growth was noticed. After incubation, each plate was scrutinized and measured for complete zones of inhibition. The zone of inhibition was measured using 
sliding calipers to the nearest whole millimeter $(\mathrm{mm})$. All the target compounds were screened for their antifungal activity against two pathogenic fungi, Fusarium oxysporum and Aspergillus niger by the poison plate technique [26].

Docking studies: The binding modes of synthesized compounds with a protein have been explained by molecular docking. All the final targets were docked individually by using Autodock 4.2 software $[27,28]$. After receiving the modeled three dimensional structure of nitrate reductase protein to Autodock 4.2 , it was structurally optimized by adding hydrogen to protein designated with kollaman charges. The model was then saved in PDBQT format after adding hydrogen. Later the ligands were prepared by adjusting the torsion angles and saved them in PDBQT format. Potential binding sites for all the proteins was selected by using PDBSUM [29]. Around binding site of protein, a grid was generated to identify the xyz coordinates. Lamarckian Genetic Algorithm (LGA) was selected for docking, freezing and default parameters used in autodock4.2. The structural intercommunications between PDB with two inhibitors were docked separately. The binding modes of protein and their binding energies of ligands were commonly estimated by performing Docking studies. X,Y,Z coordinates of PDB were selected by using SPDBV.

\section{RESULTS AND DISCUSSION}

Antibacterial activity: By considering streptomycin as a standard drug, the in vitro antibacterial studies of newly synthesized compounds were assessed against two Grampositive strains and two Gram-negative bacteria (Table-1). The antibacterial activity of the final compounds varied from moderate to excellent over the bacterial strains used. But some of the target compounds did not show activity.
From the above results, it can be understood that the compound $\mathbf{4 c}$ was found to exhibit excellent activity against Bacillus subtilis B29 and Staphylococcus aureus S276 and also shows good activity against $P$. aeruginosa ATCC 15442 , Escherichia coli E266. A capable activity against all the bacterial strains has been exhibited by Imidazole-pyrazoles containing electron donor properties. An increased zone of inhibition 21, 20 and $16 \mathrm{~mm}$ has been exhibited by compounds $\mathbf{4 c}, \mathbf{4 f}$ and $\mathbf{4 g}$, respectively when compared to the standard drug streptomycin (18 mm) against Bacillus subtilis B29 strain. Compounds $\mathbf{4 f}(19 \mathrm{~mm})$ and $\mathbf{4 g}(19 \mathrm{~mm})$ showed a good activity against the Gram-negative bacteria, Escherichia coli E266. The antifungal screening results of all the compounds were compared with standard antifungal drug, the fluconazole and observed that all the compounds have showed moderate activity. The activity of compounds $\mathbf{4 a}, \mathbf{4 c}$ against Fusarium oxysporum was slightly higher with zone of inhibition $13,12 \mathrm{~mm}$, respectively. Target compounds $\mathbf{4 h}$, $\mathbf{4 a}$ shows very good antifungal activity against $A$. niger strain with a zone of inhibition $15 \mathrm{~mm}$.

Docking: Docking studies of all the synthesized compounds into the binding site of the proteins was performed to determine the binding accord of the complex. It is a significant part of the structure based drug design process "crystal structure of the $\mathrm{C}(30)$ carotenoid dehydrosqualene synthase from Staphylococcus aureus complexed with bisphosphonate BPH700" (2ZCS). Molecular docking studies was most extensively used method for the calculation of protein-ligand interactions and all the synthesized compounds are summarized in Table2. In this study, some of the new ligands (6) have identified as potent antibacterial inhibitors from binding free energy assessment to assign the best binding conformation by AutoDock4. 2 version and it is an efficient method to predict the potential

TABLE-1

ANTIBACTERIAL, ANTIFUNGAL ACTIVITY OF SYNTHESIZED COMPOUNDS 4a-h

\begin{tabular}{|c|c|c|c|c|c|c|}
\hline \multirow{3}{*}{ Compound No. } & \multicolumn{6}{|c|}{ Zone of inhibition in $\mathrm{mm}$ at $25 \mu \mathrm{g} / \mathrm{mL}$} \\
\hline & \multicolumn{2}{|c|}{ Gram-positive bacteria } & \multicolumn{2}{|c|}{ Gram-negative bacteria } & \multicolumn{2}{|c|}{ Fungal strains } \\
\hline & $\begin{array}{c}\text { Bacillus subtilis } \\
\text { B29 }\end{array}$ & $\begin{array}{c}\text { Staphylococcus } \\
\text { aureus S276 }\end{array}$ & $\begin{array}{c}P . \text { aeruginosa } \\
\text { ATCC } 15442\end{array}$ & $\begin{array}{c}\text { Escherichia coli } \\
\text { E266 }\end{array}$ & $\begin{array}{c}\text { Fusarium } \\
\text { oxysporum }\end{array}$ & $\begin{array}{c}\text { Aspergillus } \\
\text { niger }\end{array}$ \\
\hline $\mathbf{4 a}$ & 14 & 16 & 12 & 13 & 13 & 15 \\
\hline $4 b$ & 12 & 13 & 15 & 10 & - & 12 \\
\hline $4 c$ & 21 & 20 & 18 & 15 & 12 & 14 \\
\hline $4 d$ & 13 & - & 12 & 08 & 10 & 08 \\
\hline $4 e$ & 09 & 08 & 11 & - & 06 & 13 \\
\hline $\mathbf{4 f}$ & 20 & 17 & 18 & 19 & 10 & 09 \\
\hline $4 \mathrm{~g}$ & 16 & 18 & 14 & 19 & 07 & 10 \\
\hline $4 h$ & - & 15 & 17 & 14 & 12 & 15 \\
\hline Streptomycin & 18 & 15 & 18 & 20 & - & - \\
\hline Flucanazole & - & - & - & - & 15 & 18 \\
\hline
\end{tabular}

TABLE-2

MOLECULAR DOCKING INTERACTIONS OF SOME OF THE DERIVATIVES WITH THEIR ANTIBACTERIAL PROTEIN

\begin{tabular}{|c|c|c|c|c|c|}
\hline Compd. No. & PDB & Interacting amino acids & Grid $\mathrm{X}-\mathrm{Y}-\mathrm{Z}$ coordinates & $\begin{array}{c}\text { Binding energy } \Delta \mathrm{G} \\
(\mathrm{Kcal} / \mathrm{mol})\end{array}$ & $\begin{array}{c}\text { Dissociation constant } \\
(\mathrm{KI})(\mu \mathrm{M})\end{array}$ \\
\hline $4 \mathbf{a}$ & $2 \mathrm{ZCS}$ & Asp27, Lys273 & $56.026,5.999,60.238$ & -6.34 & 22.68 \\
\hline $4 c$ & $2 \mathrm{ZCS}$ & Asp49, Arg265 & $56.026,5.999,60.238$ & -6.24 & 26.47 \\
\hline $4 e$ & $2 \mathrm{ZCS}$ & Lys273, Asp27 & $56.026,5.999,60.238$ & -7.08 & 6.47 \\
\hline $4 f$ & $2 \mathrm{ZCS}$ & Asp27, Lys273 & $56.026,5.999,60.238$ & -5.77 & 58.77 \\
\hline $4 \mathrm{~g}$ & $2 \mathrm{ZCS}$ & Lys 273 & $56.026,5.999,60.238$ & -7.89 & 1.66 \\
\hline $4 h$ & $2 \mathrm{ZCS}$ & Lys 273 & $56.026,5.999,60.238$ & -7.48 & 3.27 \\
\hline Streptomycin & $2 \mathrm{ZCS}$ & Ser21 & $56.026,5.999,60.238$ & -5.85 & 51.74 \\
\hline
\end{tabular}



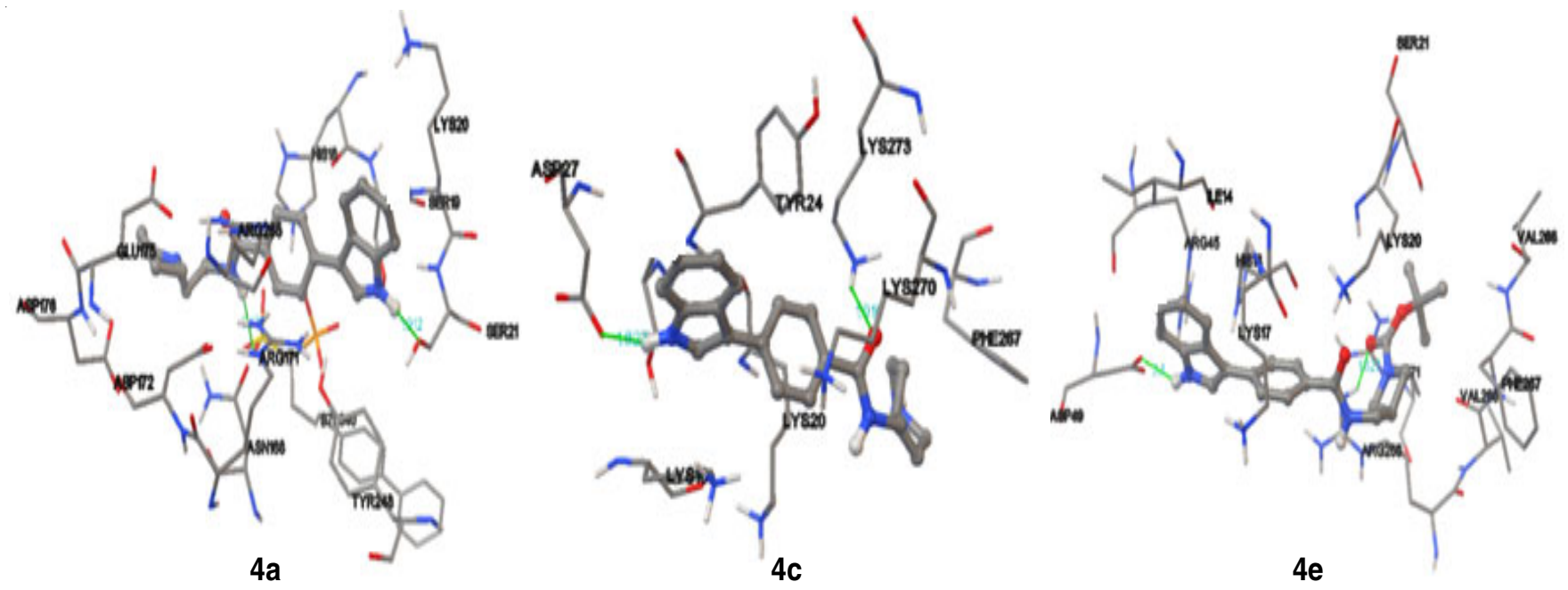

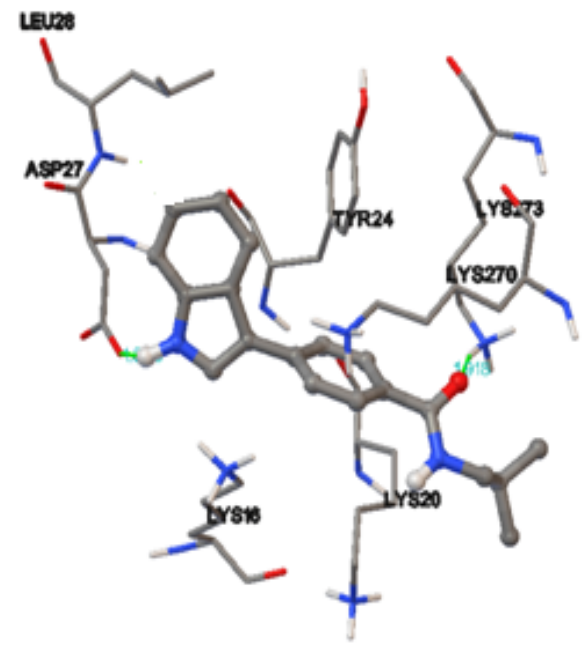

$4 f$

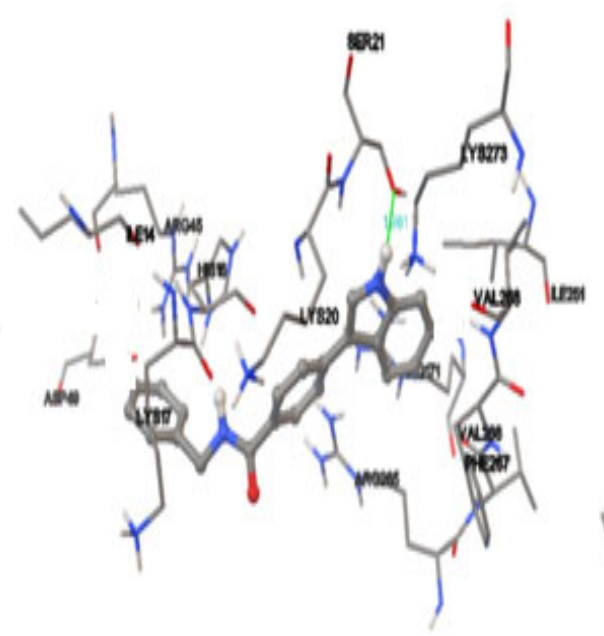

$4 g$

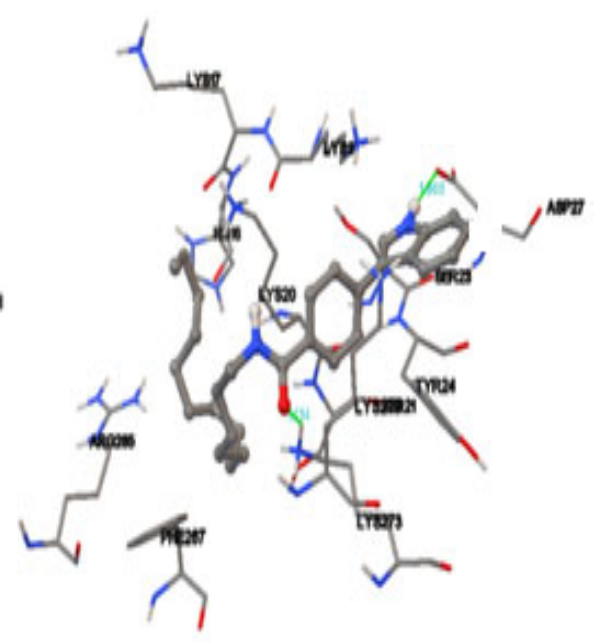

$4 \mathrm{~h}$

Fig. 1. Docking conformations complexed with bisphosphonate BPH-700 (2ZCS)

ligand interactions. Experimental activities and predicted values by Lamarckian Genetic Algorithm and the derivatives selected for molecular docking have some collective structural features.

The compound $4 \mathbf{g}$ shows highest binding energy with amino acid interactions LYS273 with binding energy $\Delta \mathrm{G}=$ -7.89 and dissociation constant in $1.66 \mu \mathrm{M}$. Fig. 1 illustrates some of the synthesized compounds $4 \mathrm{~h}$ exhibited binding energy 7.48 and 3.27 $\mu \mathrm{M}$ values and interactions with LYS273. Furthermore, the targets $\mathbf{4 a}, \mathbf{4 c}, \mathbf{4 e}, \mathbf{4 f}$ effective interactions with two amino acids Asp27, Lys273, Asp49, Arg265, with binding energies $-6.34,-6.24,7.08,5.77 \mathrm{Kcal} / \mathrm{Mol}$ values, respectively. The docking score and $\mathrm{H}$-bond interactions, van der Waal forces were done for all the synthesized compounds used in this study (green colour lines). Binding energies were measured, it consists of van der Waal forces, hydrogen bondings, $\pi-\pi$ interactions, cation- $\pi$ interactions, etc. and the hydrogen bonding distance of all the molecules with proteins is less than $2.0 \AA$.

\section{CONFLICT OF INTEREST}

The authors declare that there is no conflict of interests regarding the publication of this article.

\section{REFERENCES}

1. R.H. Wiley, eds.: L.C. Behr, R. Fusco and C.H. Jarboe, The Chemistry of Heterocyclic Chemistry: Pyrazoles, Pyrazolines, Pyrazolidines, Indazoles and Condensed Rings, Interscience Publishers: John Wiley \& Sons, pp 3-20 (1967).

2. G.M. Badger, The Chemistry of the Heterocyclic Compounds. Academic Press: New York and London, pp. 5-6 (1961).

3. A.K. Tewari and A. Mishra, Bioorg. Med. Chem., 9, 715 (2001); https://doi.org/10.1016/S0968-0896(00)00285-6.

4. J.S. Larsen, M.A. Zahran, E.B. Pedersen and C. Nielsen, Monatsh. Chem., 130, 1167 (1999);

https://doi.org/10.1007/PL00010295.

5. E.V. Pimenova and É.V. Voronina, Pharm. Chem. J., 35, 602 (2001); https://doi.org/10.1023/A:1015141710100.

6. I. Bouabdallah, L.A. M'Barek, A. Zyad, A. Ramdani, I. Zidane and A. Melhaoui, Nat. Prod. Res., 20, 1024 (2006);

https://doi.org/10.1080/14786410600921441.

7. H.J. Park, K. Lee, S.J. Park, B. Ahn, J.C. Lee, H.Y. Cho and K.I. Lee, Bioorg. Med. Chem. Lett., 15, 3307 (2005); https://doi.org/10.1016/j.bmcl.2005.03.082.

8. C.K. Chu and S.J. Cutler, Heterocycl. Chem., 23, 289 (1986); https://doi.org/10.1002/jhet.5570230201.

9. V. Michon, C.H. du Penhoat, F. Tombret, J.M. Gillardin, F. Lepage and L. Berthon, Eur. J. Med. Chem., 30, 147 (1995); https://doi.org/10.1016/0223-5234(96)88220-1.

10. M.J. Genin, C. Biles, B.J. Keiser, S.M. Poppe, S.M. Swaney, W.G. Tarpley, Y. Yagi and D.L. Romero, J. Med. Chem., 43, 1034 (2000); https://doi.org/10.1021/jm990383f. 
11. Y.R. Huang and J.A. Katzenellenbogen, Org. Lett., 2, 2833 (2000); https://doi.org/10.1021/ol0062650.

12. S.R. Stauffer, C.J. Coletta, R. Tedesco, G. Nishiguchi, K. Carlson, J. Sun, B.S. Katzenellenbogen and J.A. Katzenellenbogen, J. Med. Chem., 43, 4934 (2000); https://doi.org/10.1021/jm000170m.

13. S. Ruiu, G.A. Pinna, G. Marchese, J.M. Mussinu, P. Saba, S. Tambaro, P. Casti, R. Vargiu and L. Pani, J. Pharmacol. Exp. Ther., 306, 363 (2003); https://doi.org/10.1124/jpet.103.049924.

14. R. Olivera, R. Sanmartin and E. Dominguez, J. Org. Chem., 65, 7010 (2000); https://doi.org/10.1021/jo000609i.

15. The Merck Index, Merck \& Co Inc., edn 13, p. 1785 (2001).

16. M. Amari, M. Fodili, B. Nedjar-kolli, A.É.P. Hoffmann and J. PÉriÉ, J. Heterocycl. Chem., 39, 811 (2002); https://doi.org/10.1002/jhet.5570390429.

17. P. Kohler, Int. J. Parasitol., 31, 336 (2001); https://doi.org/10.1016/S0020-7519(01)00131-X.

18. A.T.S. Mavrova, K.K. Anichina, D.I. Vuchev, J.A. Tsenov, M.S. Kondeva and M.K. Micheva, Bioorg. Med. Chem., 13, 5550 (2005); https://doi.org/10.1016/i.bmc.2005.06.046.

19. H. Goker, C. Kus, D.W. Boykin, S. Yildiz and N. Altanlar, Bioorg. Med. Chem., 10, 2589 (2002); https://doi.org/10.1016/S0968-0896(02)00103-7.

20. H. Göker, S. Özden, S. Yildiz and D.W. Boykin, Eur. J. Med. Chem., 40, 1062 (2005); https://doi.org/10.1016/j.ejmech.2005.05.002.
21. M. Andrzejewska and M.L. Yepez, Eur. J. Med. Chem., 37, 973 (2002); https://doi.org/10.1016/S0223-5234(02)01421-6.

22. S. Özden, D. Atabey, S. Yildiz and H. Göker, Bioorg. Med. Chem., 13, 1587 (2005); https://doi.org/10.1016/j.bmc.2004.12.025.

23. M.M. Ramla, M.A. Omar, A.-M.M. El-Khamry and H.I. El-Diwani, Bioorg. Med. Chem., 14, 7324 (2006); https://doi.org/10.1016/j.bmc.2006.06.033.

24. M. Boiani and M. Gonzalez, Mini Rev. Med. Chem., 5, 409 (2005); https://doi.org/10.2174/1389557053544047.

25. C. Valgas, S.M. Souza, E.F.A. Smânia and A. Smânia Jr, Braz. J. Microbiol., 38, 369 (2007); https://doi.org/10.1590/S1517-83822007000200034.

26. L. Rivillas-Acevedo and M. Soriano-García, J. Mex. Chem. Soc., 51, 136 (2007).

27. D.S. Goodsell and A.J. Olson, Proteins, 8, 195 (1990); https://doi.org/10.1002/prot.340080302.

28. G.M. Morris, R. Huey, W. Lindstrom, M.F. Sanner, R.K. Belew, D.S. Goodsell and A.J. Olson, J. Comput. Chem., 30, 2785 (2009); https://doi.org/10.1002/jcc.21256.

29. R.A. Laskowski, Nucleic Acids Res., 37, 355 (2009); https://doi.org/10.1093/nar/gkn860. 